\title{
A New Method for Designing Causal Stable IIR Variable Fractional Delay Digital Filters
}

\author{
K. M. Tsui, S. C. Chan, Member, IEEE, and H. K. Kwan, Senior Member, IEEE
}

\begin{abstract}
This paper studies the design of causal stable Farrowbased infinite-impulse response (IIR) variable fractional delay digital filters (VFDDFs), whose subfilters have a common denominator. This structure has the advantages of reduced implementation complexity and avoiding undesirable transient response when tuning the spectral parameter in the Farrow structure. The design of such IIR VFDDFs is based on a new model reduction technique which is able to incorporate prescribed flatness and peak error constraints to the IIR VFDDF under the second order cone programming framework. Design example is given to demonstrate the effectiveness of the proposed approach.
\end{abstract}

Index Terms-Constrained model reduction, delay filter, infiniteimpulse response (IIR), variable digital filter (VDF).

\section{INTRODUCTION}

V ARIABLE fractional delay digital filters (VFDDFs) find important applications in signal processing and communications [1]-[3]. They are useful to the implementation of arbitrary sample rate converters [4], digital synchronizers [5] and other related applications. VFDDFs are also a kind of variable digital filters (VDFs) where online tuning of frequency characteristics is required. A number of useful methods for designing Farrow-based finite-impulse response (FIR) VDFs, namely weighted least squares [2], [6], linear programming [7], semidefinite programming [4] and second order cone programming (SOCP) [8], have been proposed. The latter two approaches are able to design both linear-phase and low-delay FIR VDFs with either minimax or LS criterion subject to wide variety of constraints.

IIR VDFs, on the other hand, have also received considerable attention for their potential advantages of lower system delay, sharper cutoff and higher stopband attenuation over their FIR counterparts. However, the design of IIR VDFs is usually complicated by the highly nonlinear objective functions and stability constraints [3], [9]-[12]. Therefore, most conventional methods mainly focus on how the stability can be guaranteed. For the design of IIR VFFDFs, [3] and [10] proposed to employ allpass filters to simplify the design problem. Also, they mentioned that due to the unit magnitude of allpass filters, the available degree of freedom will be reduced as compared with general IIR filters.

Manuscript received April 4, 2007; revised June 8, 2007. This paper was recommended by Associate Editor H. Johansson.

K. M. Tsui and S. C. Chan are with the Department of Electrical and Electronic Engineering, The University of Hong Kong, Pokfulam, Hong Kong (e-mail: kmtsui@eee.hku.hk; scchan@eee.hku.hk).

H. K. Kwan is with the Department of Electrical Engineering, University of Windsor, Windsor, ON N9B 3P4, Canada (e-mail: kwan1@uwindsor.ca).

Digital Object Identifier 10.1109/TCSII.2007.903784
More recently, an optimization method for designing VFDDFs using general IIR filters was proposed in [12]. All these approaches assume that the numerator and denominator or the poles and zeros of the IIR VFDDFs are functions of the spectral parameter. This structure will however suffer from undesirable transient responses because the states of the IIR VFDDFs are abruptly changed during the parameter tuning process [13]. Another useful method proposed in [6] for designing IIR VDFs is based on model reduction of FIR prototype filters. In addition to its simple design procedure, the advantage of the model reduction approach is that the resulting IIR filter is guaranteed to be stable, and the frequency characteristics is well preserved. However, it does not allow precise control of the frequency response and incorporation of other constraints, such as prescribed number of zeros and peak error constraints.

In this paper, we study the design problem of causal stable IIR VFDDFs whose subfilters have a common denominator. This structure can avoid undesirable transient response during parameter tuning and reduce the implementation complexity. Moreover, a new design method for IIR VFDDFs using a new constrained model reduction technique is proposed. This method, which can be viewed as a modification of the model reduction technique proposed in [14], has been successfully applied to the design of casual stable IIR filters [15] with prescribed flatness and peak error constraints.

Important advantages of the proposed method are that the numerator and denominator are determined separately and the stability of the model-reduced filter is guaranteed. More precisely, the common denominator is first obtained by model-reducing an average response of the prototype FIR VFDDF. The numerator is then designed using SOCP so that prescribed flatness and peak error constraints can be imposed to improve the frequency characteristics of the final IIR VFDDF. Prescribed flatness constraints like multiple zeros in the stopband are sometimes desirable in sample rate converters for the suppression of alias components, while peak error constraints are useful to limit the sidelobes and undesirable peaks in filters with wide unconstrained transition band and low system delay. Owing to the improved frequency characteristics of the proposed IIR VFDDFs, further optimization is usually not required. Design results show that the proposed IIR VFDDFs have better performances and lower implementation complexity than the conventional methods in [6] and [12]. The paper is organized as follows: Sections II and III are devoted to the proposed IIR VFDDF structure and its design using the constrained model reduction technique. Design example is given in Section IV. Finally, conclusion is drawn in Section IV. 


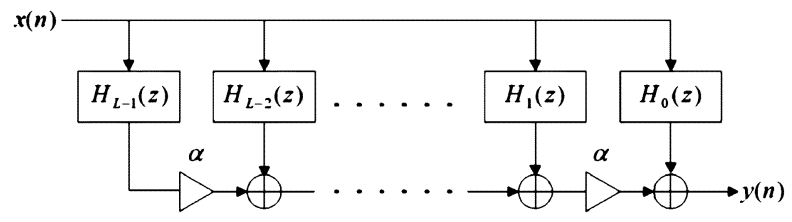

Fig. 1. Farrow structure for implementing a VDF.

\section{THE StRUCTURE OF IIR VFDDF}

In a VDF, the desired response $H_{d}(\omega, \alpha)$ is a function of a spectral parameter $\alpha$ (also known as tuning or control parameters). The spectral characteristics of a desired VDF can therefore be continuously varied by changing the parameter $\alpha$.

The impulse response of the VDF under consideration is assumed to be $h(n, \alpha)=\sum_{l=0}^{L-1} h_{l}(n) \alpha^{l}$, where $\alpha$ is assumed to vary linearly over a finite interval $\left[\alpha_{\min }, \alpha_{\max }\right]$. The z-transform of the VDF can then be written as

$$
H(z, \alpha)=\sum_{n=-\infty}^{\infty} h(n, \alpha) z^{-n}=\sum_{n=-\infty}^{\infty} \sum_{l=0}^{L-1} h_{l}(n) \alpha^{l} z^{-n} .
$$

Interchanging the order of summation, one obtains

$$
H(z, \alpha)=\sum_{l=0}^{L-1}\left[\sum_{n=-\infty}^{\infty} h_{l}(n) z^{-n}\right] \cdot \alpha^{l}=\sum_{l=0}^{L-1} H_{l}(z) \cdot \alpha^{l}
$$

where $H_{l}(z)=\sum_{n=-\infty}^{\infty} h_{l}(n) z^{-n}$ is the $l$ th subfilter, which can either be FIR or IIR function. (1) suggests a very useful structure for implementing VDF called the Farrow's structure [1] which is shown in Fig. 1. For FIR VDF, the $l$ th subfilter is given by $H_{l}(z)=\sum_{n=0}^{N-1} h_{l}(n) z^{-n}$, where $N$ is the filter length.

A similar structure was applied in [3], [9]-[12] for IIR VDFs, where the poles and zeros of IIR subfilters are assumed to be polynomials of the spectral parameters. Unlike FIR VDFs, the design of IIR VDFs requires nonlinear optimization, which is rather time consuming. Another important problem of IIR VDFs with direct tuning of the poles and zeros is the undesirable transient response generated during parameter tuning [13].

In VFDDF, its desired response can be written as

$$
H_{d}(\omega, \alpha)= \begin{cases}e^{-j \omega \tau(\alpha)}, & \omega \in\left[0, \omega_{p}\right] \\ 0, & \omega \in\left[\omega_{s}, \pi\right]\end{cases}
$$

where $\tau(\alpha)=\tau+\alpha$ is the group delay of the VFDDF and it can be varied linearly by the spectral parameter $\alpha ; \omega_{p}$ and $\omega_{s}$ are respectively the passband and stopband cutoff frequencies. In this paper, we shall consider the design of causal stable IIR VFDDF whose subfilters have a common denominator. More precisely, the proposed IIR VFDDF can be expressed as

$$
\begin{aligned}
\hat{H}(z, \alpha) & =P(z, \alpha) / Q(z) \\
& =\left(\sum_{l=0}^{L-1} P_{l}(z) \cdot \alpha^{l}\right) / Q(z)
\end{aligned}
$$

where $P_{l}(z)=\sum_{n=0}^{N_{p}-1} p_{l}(n) z^{-n}, Q(z)=\sum_{n=0}^{N_{q}-1} q(n) z^{-n}$ $(q(0)=1)$ and $N_{p} \geqslant N_{q}$. Fig. 2 shows the structure of the proposed IIR VFDDF. An advantage of this structure is that it does not suffer from the problem of undesirable transient response which is generated when the parameter $\alpha$ is varied (except at

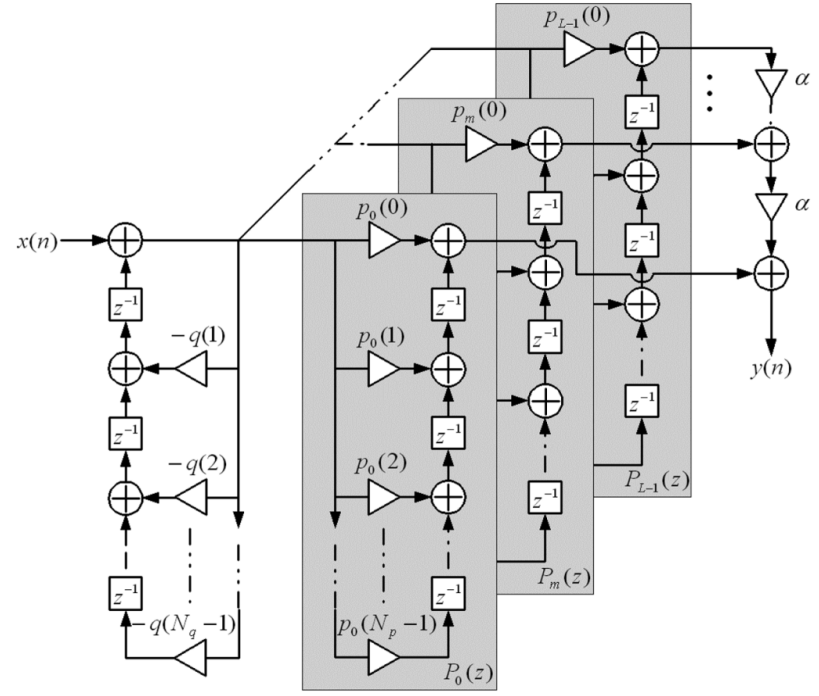

Fig. 2. Proposed structure of IIR VFDDF.

the very beginning). It is because the subfilters' outputs are directly combined to form the desired output without changing their internal states. Also, the arithmetic complexity is significantly reduced as all the subfilters have the same denominator. Furthermore, the numerator and denominator coefficients can be simultaneously implemented in a multiplier block for efficient multiplier-less realization [16].

\section{DESIGN OF IIR VFDDF}

Suppose that we have designed a FIR VFDDF in the form of (1) using any existing methods for a given specification. To design IIR VFDDFs in (3), we employ the model reduction method in [15], which can approximate an arbitrary FIR function by an IIR function. The advantage is that the denominator of the IIR function can be found without the knowledge of numerator, unlike other model reduction techniques. Based on this observation, we propose to design the numerators $P_{l}(z)$ using SOCP, where additional constraints can be incorporated to improve the performance, after $Q(z)$ has been found. We now describe the procedures for determining $Q(z)$ and $P_{l}(z)$.

\section{A. Determination of Denominator}

In [15], the denominator of the model-reduced IIR filter can be determined using a simple iterative procedure, given a FIR function. To use this technique, we assume that $\hat{H}(z, \alpha)$ has the same denominator $Q(z)$ as in (3). A good method to determine $Q(z)$ is to model-reduce the average response of $H(z, \alpha)$ over the whole tuning range as follows:

$$
\begin{aligned}
F(z) & =\left[\int_{\alpha_{\min }}^{\alpha_{\max }}\left(\sum_{l=0}^{L-1} H_{l}(z) \cdot \alpha^{l}\right) d \alpha\right] /\left(\alpha_{\max }-\alpha_{\min }\right) \\
& =\sum_{l=0}^{L-1} \frac{\left(\alpha_{\max }^{l+1}-\alpha_{\min }^{l+1}\right) H_{l}(z)}{\left(\alpha_{\max }-\alpha_{\min }\right)(l+1)}
\end{aligned}
$$

The main reason for considering $F(z)$ is that the magnitude response of $H(z, \alpha)$ does not change significantly with $\alpha$ in the passband. This also explains why a common denominator 
is considered because it mainly contributes to the sharp change in the magnitude response near the passband edge. Denote the polynomial which approximates $Q(z)$ at the $k$ th iteration by $Q^{(k)}(z)=1+\sum_{n=1}^{L_{q}-1} q^{(k)}(n) z^{-n}$, where $Q^{(0)}(z)=1$. By defining

$$
X^{(k)}(z)=z^{-(L-1)} F\left(z^{-1}\right) / Q^{(k-1)}(z)=\sum_{n=0}^{\infty} x^{(k)}(n) z^{-n}
$$

$q^{(k)}(n)$ can be obtained by minimizing

$$
C^{(k)}=\left(\boldsymbol{B}^{(k)} \boldsymbol{q}^{(k)}-\boldsymbol{d}^{(k)}\right)^{T} \cdot\left(\boldsymbol{B}^{(k)} \boldsymbol{q}^{(k)}-\boldsymbol{d}^{(k)}\right)
$$

where

$$
\begin{aligned}
& \boldsymbol{B}^{(k)}=\left[\begin{array}{cccc}
x^{(k)}(0) & 0 & \cdots & 0 \\
x^{(k)}(1) & x^{(k)}(0) & \ddots & \vdots \\
\vdots & \vdots & \ddots & 0 \\
x^{(k)}\left(L_{q}-2\right) & x^{(k)}\left(L_{q}-3\right) & \cdots & x^{(k)}(0) \\
\vdots & \vdots & & \vdots \\
x^{(k)}(L-2) & x^{(k)}(L-3) & \cdots & x^{(k)}\left(L-L_{q}\right)
\end{array}\right] \\
& \boldsymbol{q}^{(k)}=\left[q^{(k)}\left(L_{q}-1\right), \ldots, q^{(k)}(1)\right]^{T} \quad \text { and } \\
& \boldsymbol{d}^{(k)}=-\left[0, \ldots, 0, x^{(k)}(0), \ldots, x^{(k)}\left(L-L_{q}-1\right)\right]^{T} .
\end{aligned}
$$

The basic idea is to find $\boldsymbol{q}^{(k)}$ such that $C^{(k)}$ is the smallest among all the iterations for a sufficiently large $k$. More importantly, the roots of the resulting $Q^{(k)}(z)$, which minimizes $C^{(k)}$ for an arbitrarily given $X^{(k)}(z)$, are proved to lie strictly inside the unit circle, and thus $\hat{H}(z, \alpha)$ is always stable. Interested readers are referred to [14] for more details.

\section{B. Determination of Numerator Using SOCP}

Once the common denominator $Q\left(e^{j \omega}\right)$ is designed, $\hat{H}\left(e^{j \omega}, \alpha\right)$ in (3) can be rewritten more compactly in matrix form in terms of the design variables $\boldsymbol{p}_{l}=\left[p_{l}(0), \ldots, p_{l}\left(N_{p}-\right.\right.$ $1)]^{T}$, for $l=0,1, \ldots, L-1$, as follows:

$$
H\left(e^{j \omega}, \alpha\right)=\boldsymbol{a}^{T}\{\boldsymbol{c}(\omega, \alpha)-j \boldsymbol{s}(\omega, \alpha)\}
$$

where $\boldsymbol{a}=\left[\boldsymbol{p}_{0}^{T}, \ldots, \boldsymbol{p}_{L-1}^{T}\right]^{T}, \boldsymbol{c}(\omega, \alpha)=\operatorname{Re}\left[\boldsymbol{e}_{1}(\omega, \alpha) / Q\left(e^{j \omega}\right)\right]$, $\boldsymbol{s}(\omega, \alpha)=\operatorname{Im}\left[\boldsymbol{e}_{1}(\omega, \alpha) / Q\left(e^{j \omega}\right)\right] ; \boldsymbol{e}_{1}(\omega, \alpha)=\left[\boldsymbol{e}(\omega)^{T}, \alpha^{1} \cdot \boldsymbol{e}(\omega)^{T}\right.$ $\left., \ldots, \alpha^{L-1} \cdot \boldsymbol{e}(\omega)^{T}\right]^{T}, \boldsymbol{e}(\omega)=\left[1, e^{-j \omega}, \ldots, e^{-j\left(N_{p}-1\right) \omega}\right]^{T}$, $\operatorname{Re}[x]$ and $\operatorname{Im}[x]$ denote the real and imaginary parts of $x$, respectively.

First of all, we approximate the desired response $H_{d}(\omega, \alpha)$ in (2) by $H(z, \alpha)$ in the minimax sense by minimizing

$$
E_{\infty}=\max _{(\omega, \alpha) \in \Omega}\left\{W(\omega, \alpha)\left|H\left(e^{j \omega}, \alpha\right)-H_{d}(\omega, \alpha)\right|\right\}
$$

where $W(\omega, \alpha)$ is a positive weighting function, and $\Omega$ is the (frequency, tuning range) of interest. The minimization problem of $E_{\infty}$ in (8) can be reformulated as

$$
\min _{\boldsymbol{a}} \delta \text { subject to } \delta-\left[\gamma_{R}^{2}(\omega, \alpha)+\gamma_{I}^{2}(\omega, \alpha)\right]^{1 / 2} \geqslant 0
$$

where $\gamma_{R}(\omega, \alpha)=W(\omega, \alpha)\left\{\boldsymbol{a}^{T} \boldsymbol{c}(\omega, \alpha)-\operatorname{Re}\left[H_{d}(\omega, \alpha)\right]\right\}$ and $\gamma_{I}(\omega, \alpha)=W(\omega, \alpha)\left\{\boldsymbol{a}^{T} \boldsymbol{s}(\omega, \alpha)+\operatorname{Im}\left[H_{d}(\omega, \alpha)\right]\right\},(\omega, \alpha) \in \Omega$. Discretizing the frequency variable $\omega$ and spectral parameter $\alpha$ over a dense set of frequencies $\left\{\omega_{i}, 1 \leqslant i \leqslant K_{1}\right\}$ and spectral parameters $\left\{\alpha_{k}, 1 \leqslant k \leqslant K_{2}\right\}$ in the range of interests, where $K_{1}$ and $K_{2}$ are positive integers, the constraints in (9) become $\delta-\left[\alpha_{R}^{2}\left(\omega_{i}, \alpha_{k}\right)+\alpha_{I}^{2}\left(\omega_{i}, \alpha_{k}\right)\right]^{1 / 2} \geqslant 0$. Moreover, by defining the augmented variable $\boldsymbol{x}=\left[\begin{array}{ll}\delta & \boldsymbol{a}^{T}\end{array}\right]^{T}$, (9) can be cast to the standard SOCP problem as follows:

$$
\min _{\boldsymbol{x}} \boldsymbol{c}^{T} \boldsymbol{x} \text { subject to } \boldsymbol{c}^{T} \boldsymbol{x} \geqslant\left\|\boldsymbol{F}_{i, k} \boldsymbol{x}-\boldsymbol{g}_{i, k}\right\|_{2}
$$

where $\boldsymbol{c}=\left[\begin{array}{ll}1 & \boldsymbol{O}_{N_{p} \times L}^{T}\end{array}\right]^{T}, \boldsymbol{F}_{i, k}=W\left(\omega_{i}, \alpha_{k}\right) \cdot\left[\begin{array}{cc}0 & \boldsymbol{c}\left(\omega_{i}, \alpha_{k}\right)^{T} \\ 0 & \boldsymbol{s}\left(\omega_{i}, \alpha_{k}\right)^{T}\end{array}\right]$ and $\boldsymbol{g}_{i, k}=W\left(\omega_{i}, \alpha_{k}\right) \cdot\left[H_{R_{-} d}\left(\omega_{i}, \alpha_{k}\right)-H_{I_{-} d}\left(\omega_{i}, \alpha_{k}\right)\right]^{T} ; \boldsymbol{O}_{D}$ is a $D \times 1$ zero vector.

Instead of using the minimax criterion, the following least squares design criterion can be minimized:

$$
E_{\mathrm{LS}}=\int_{\Omega} W(\omega, \alpha) \cdot\left|H\left(e^{j \omega}, \alpha\right)-H_{d}(\omega, \alpha)\right|^{2} d \omega d \alpha
$$

$E_{\mathrm{LS}}$ can also be written as a quadratic function of $\boldsymbol{a}$ as

$$
\begin{aligned}
E_{\mathrm{LS}} & =\boldsymbol{a}^{T} \boldsymbol{U} \mathrm{a}-2 \boldsymbol{a}^{T} \boldsymbol{g}+b \\
& =\left|\boldsymbol{U}^{1 / 2} \boldsymbol{a}-\boldsymbol{U}^{-1 / 2} \boldsymbol{g}\right|_{2}-\boldsymbol{g}^{T} \boldsymbol{U}^{-1} \boldsymbol{g}+b
\end{aligned}
$$

where $\boldsymbol{U}=\int_{\Omega} W(\omega, \alpha) \cdot\left[\boldsymbol{c}(\omega, \alpha) \boldsymbol{c}(\omega, \alpha)^{T}+\boldsymbol{s}(\omega, \alpha) \boldsymbol{s}(\omega, \alpha)^{T}\right]$ $d \omega d \alpha, \boldsymbol{g}=\int_{\Omega} W(\omega, \alpha)\left[\boldsymbol{c}(\omega, \alpha) H_{R_{-} d}(\omega, \alpha)+\boldsymbol{s}(\omega, \alpha)\right.$ $\left.H_{I_{-} d}(\omega, \alpha)\right] d \omega d \alpha$, and $b=\int_{\Omega} W(\omega, \alpha)\left|H_{d}(\omega, \alpha)\right|^{2} d \omega d \alpha$. The minimization problem of $E_{\mathrm{LS}}$ can be formulated as the following SOCP

$$
\min _{\boldsymbol{x}} \boldsymbol{c}^{T} \boldsymbol{x} \text { subject to } \boldsymbol{c}^{T} \boldsymbol{x} \geqslant\|\overline{\boldsymbol{U}} \boldsymbol{x}-\overline{\boldsymbol{g}}\|_{2}
$$

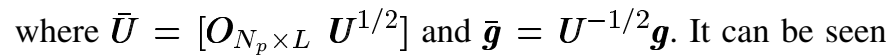
that the number of constraints in the LS design is considerably less than the minimax design criterion, which usually leads to lower design complexity. The advantage of formulating the LS design problem as a SOCP is that additional linear equalities or convex quadratic constraints can easily be incorporated to (13), as we shall illustrate in later sections.

\section{Imposing Multiple Zeros in the Stopband}

When designing digital filters, it is often required to impose certain constraints on the frequency characteristics. One commonly encountered constraint is prescribed number of zeros in the stopband, which are desirable in designing sample rate converters in order to suppress the alias components [4]. These constraints can be obtained by equating the derivatives of the design response and its ideal counterparts, and are equivalent to

$$
\left.\frac{d^{v}}{d \omega^{v}} P\left(e^{j \omega}, \alpha\right)\right|_{\omega=\bar{\omega}}=0, \quad v=0,1, \ldots, V_{\hat{\omega}_{s}}-1
$$




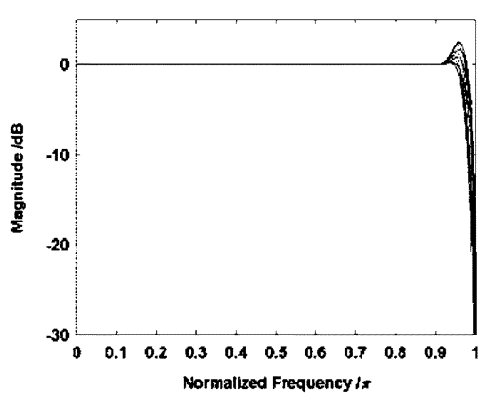

(a)

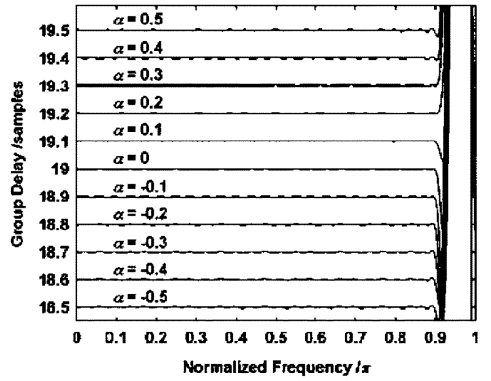

(d)

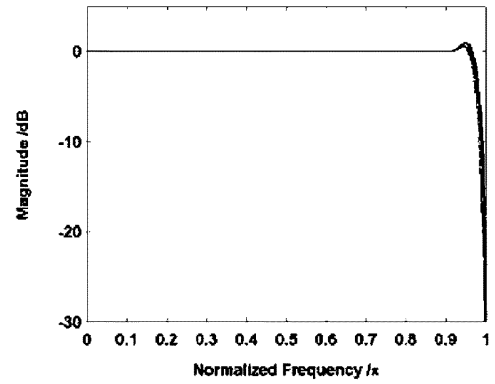

(b)

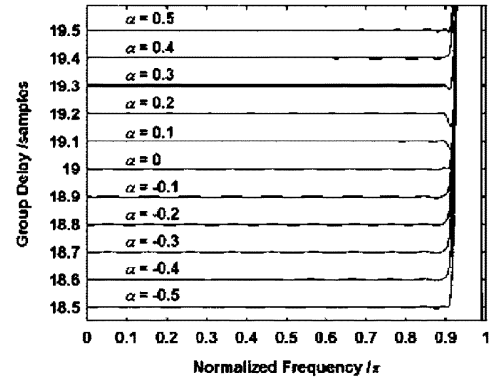

(e)

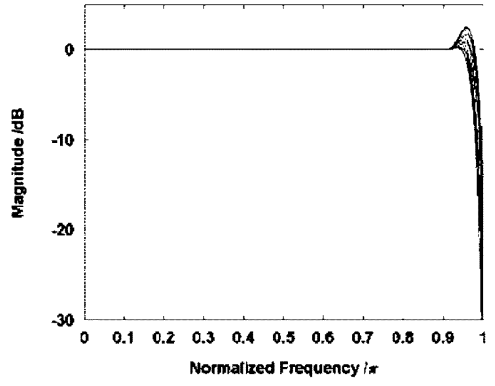

(c)

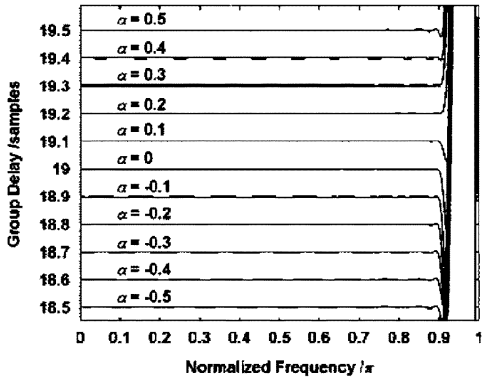

(f)

Fig. 3. (a), (d) Frequency and group delay responses of the low-delay FIR VFDDF (prototype). (b), (e) Frequency and group delay responses of the proposed IIR VFDDF. (c), (f) Frequency and group delay responses of the IIR VFDDF obtained using conventional model reduction [6].

Substituting (3) into (14), we have

$$
\sum_{n=0}^{N-1} \sum_{l=0}^{L-1} n^{v} \cdot p_{l}(n) \cdot \alpha^{l} e^{-j \hat{\omega}_{s} n}=0, \quad v=0,1, \ldots, V_{\hat{\omega}_{s}}-1 .
$$

To satisfy (15) for the entire range of the tuning parameter $\alpha$, the constraints $\sum_{n=0}^{N-1}\left[n^{v} \cdot p_{l}(n) \cdot e^{-j \hat{\omega}_{s} n}\right]=0, v=$ $0,1, \ldots, V_{\hat{\omega}_{s}}-1$ and $l=0,1, \ldots, L-1$, are imposed to each subfilter. These constraints can be written as the matrix representations

$$
\boldsymbol{A}_{l} \boldsymbol{p}_{l}=\boldsymbol{O}_{V_{\hat{\omega}_{s}}, \quad l=0,1, \ldots, L-1}
$$

where $\left[A_{l}\right]_{v, n}=n^{v} e^{-j \hat{\omega}_{s} n}$ for $v=0,1, \ldots, V_{\hat{\omega}_{s}}-1$, Here, $[\boldsymbol{A}]_{m, n}$ denotes the $(m, n)$ th entry of matrix $\boldsymbol{A}$. To incorporate them to the SOCP in (10) or (13), we combine all the constraints in (16) to form $\boldsymbol{A} a=O_{L \times V_{\hat{\omega}_{s}}}$, where $A=\operatorname{diag}\left\{\boldsymbol{A}_{0}, \ldots, \boldsymbol{A}_{L-1}\right\}$. The minimization problems in (10) and (13) can be solved subject to these linear equality constraints using SOCP.

\section{Peak Error and Convex Quadratic Constraints}

To avoid excessive sidelobes of the LS solution and/or to suppress the undesired overshoot in the transition band for lowdelay VFDDF, additional peak error constraints should be imposed to the frequency of interest. The peak error constraint can be written as $\left|\hat{H}\left(e^{j \omega}, \alpha\right)\right| \leqslant \varepsilon, \omega \in\left[\omega_{1}, \omega_{2}\right],(\omega, \alpha) \in \Omega$, where $\varepsilon$ be the peak ripple to be imposed in a frequency band $\omega \in\left[\omega_{1}, \omega_{2}\right]$ (a collection of frequency bands is also feasible). Similar to the minimax formulation, one gets

$$
\varepsilon \geqslant\|\boldsymbol{R} x\|_{2}, \quad \text { where } \boldsymbol{R}=\left[\begin{array}{ll}
0 & \boldsymbol{c}(\omega, \alpha)^{T} \\
0 & \boldsymbol{s}(\omega, \alpha)^{T}
\end{array}\right] .
$$

After discretizing $\omega$ and $\alpha$ over the range of interest, the resulting constraints on the peak ripples can be augmented to the existing constraints in (10) and (13) for the minimax and LS criterion, respectively.

\section{E. Selection of the Length of Denominator}

In order to approximate $H(z, \alpha)$ to a sufficiently small error level using the technique in [15], the length of the denominator of $\hat{H}(z, \alpha)$ should satisfy $N_{q} \geqslant\lceil\tau\rceil+1$, where $\lceil\cdot\rceil$ denotes the ceiling function. This tells us that the savings in number of multiplications and additions would be more pronounced if model reduction is applied to FIR functions with lower system delay. Here, we prefer to choose the group delay of the prototype FIR VFDDF as

$$
\tau \cong N / 4
$$

because a bump will usually appear at the transition band when the group delay is lower than this value. This choice allows us to set the system delay of $H(z, \alpha)$ as low as possible, while keeping a good frequency characteristic of $H(z, \alpha)$. Therefore, the implementation complexity and performance of $\hat{H}(z, \alpha)$ would be comparable to its linear phase FIR counterpart. However, the system delay is approximately reduced by a factor of two.

\section{DESIGN EXAMPLE}

In this example, a low-delay IIR VFDDF is designed using the proposed constrained model reduction method. The SOCP problems are solved using the Sedumi Matlab Toolbox [17]. As a comparison, the specifications of the VFDDF are the same as those in [12]. That is: number of subfilter $L=7, \omega_{p}=0.9 \pi, \omega_{s}=\pi$ and $\tau(\alpha)=[18,19](\tau=18.5$ and $\alpha=[-0.5,0.5])$. First of all, a low-delay FIR prototype VFDDF of length $N=72$ is designed in the minimax sense using the SOCP approach in [8]. One zero at $\omega=\pi$ is also 
TABLE I

Design Results of IIR VARIable Fractional Delay Digital Filters. $\left(\delta_{p}, \delta_{s}, \delta_{g}\right)$ : MAXIMUM (PASSBAND, STOPBAND, GROUP DELAY) ERROR; $O_{t}$ OVERSHOOT IN TRANSITION BAND; $T_{A}$ : TOTAL NUMBER OF ADDERS; $T_{M}$ : TOTAL NUMBER OF MUlTiPLIERS; N/A: NOT APPLICABLE

\begin{tabular}{c|c|c|c|c}
\hline \hline & FIR & Proposed & {$[\mathbf{1 2}]$} & {$[\mathbf{6}]$} \\
\hline Numerator & $72 \times 7$ & $37 \times 7$ & $52 \times 7$ & $37 \times 7$ \\
\hline Denominator & N/A & 26 & $16 \times 7$ & 37 \\
\hline$\delta_{p}\left(\times 10^{-4}\right)$ & 1.128 & 1.155 & 3.135 & 0.754 \\
\hline$\delta_{s}$ & 0.027 & 0.019 & N/A & 0.026 \\
\hline$\delta_{g}($ samples $)$ & 0.019 & 0.010 & $>0.010$ & 0.019 \\
\hline$O_{t}(\mathrm{~dB})$ & 2.54 & 1 & N/A & 2.54 \\
\hline $\max \left[p_{l}(n)\right]-\min \left[p_{l}(n)\right]$ & N/A & 18.8 & N/A & 2263.4 \\
\hline $\max [q(n)]-\min [q(n)]$ & N/A & 12.9 & N/A & 1749.2 \\
\hline$T_{A}$ & 503 & 283 & 474 & 294 \\
\hline$T_{M}$ & 510 & 290 & 481 & 301 \\
\hline \hline
\end{tabular}

imposed so that the model-reduced IIR VFDDF shown below would preserve similar characteristics as its FIR counterpart. Fig. 3(a) and (d) shows the frequency and group delay responses so obtained. From Fig. 3(a), it can be seen that the resultant FIR VFDDF has an overshoot of $2.54 \mathrm{~dB}$ in the transition band. This seems to be a fundamental limitation for the design of the low-delay FIR VFDDF with sharp cutoff.

Using the proposed model reduction method described in Section III, the above prototype filter is converted to an IIR VFDDF. Other parameters used in the proposed approach are as follows: the lengths of the numerator and denominator of the model-reduced filter are, respectively, chosen as $N_{p}=37$ and $N_{q}=26, K_{1}=37 \times 4=148$ and $K_{2}=31$. To suppress the overshoot, a peak transition band error constraint of $1 \mathrm{~dB}$ is imposed to the model-reduced filter. In addition, one zero at $\omega=\pi$ is imposed. Due to page limitation, only minimax criterion is considered. Fig. 3(b) and (e) shows the design results of the IIR VFDDF so obtained. It can be seen that both types of constraints are successfully imposed by the proposed method to the IIR VFDDF, at the expense of slightly lower performance at the unconstrained band. As shown in Table I, the proposed IIR VFDDF has better performances comparing to the one designed in [12]. Also, because of the structure in (3) where common denominator is employed, the implementation complexity of the proposed VFDDF is considerably lower. Besides, comparison with the conventional model reduction approach studied in [6] is also considered. In order for this approach to obtain a similar performance, the length of the denominator of the model-reduced filter have to be chosen as $N_{q}=37$. Furthermore, it should be noted that the dynamic range of the coefficients in the proposed VFDDF is much lower than the one in [6], making the implementation less complicated. The above results demonstrate the effectiveness of the proposed method in designing causal stable IIR VFDDFs. Table I summarizes the design results obtained using various methods.

\section{CONCLUSION}

A new method for designing causal stable IIR VFDDFs with prescribed flatness and peak error constraints using SOCPbased model reduction technique is presented. The proposed VFDDF structure has a common denominator and it reduces the implementation complexity and avoids undesirable transient response during parameter tuning. Design results show that the proposed method offers more flexibility, smaller coefficient dynamic range and better performance than the conventional methods studied in this paper.

\section{REFERENCES}

[1] C. W. Farrow, "A continuously variable digital delay element," in Proc. IEEE ISCAS, 1988, vol. 3, pp. 2641-2645

[2] W. S. Lu and T. B. Deng, "An improved weighted least-squares design for variable fractional delay FIR filters," IEEE Trans. Circuits Syst. II, Analog Digit. Signal Process., vol. 9, pp. 689-685, Sep. 1997.

[3] T. I. Laakso, V. Valimaki, M. Karjalainen, and U. K. Laine, "Splitting the unit delay, tools for fractional delay filter design," IEEE Signal Process. Mag., pp. 30-60, Jan. 1996.

[4] S. C. Chan, K. M. Tsui, K. S. Yeung, and T. I. Yuk, "Design and complexity optimization of a new digital IF for software radio receivers with prescribed output accuracy," IEEE Trans. Circuits Syst. I, Reg. Papers, vol. 54, no. 2, pp. 351-366, Feb. 2007.

[5] L. Erup, F. M. Gardner, and R. A. Harris, "Interpolation in digital modems. II. Implementation and performance,” IEEE Trans. Commun., vol. 41, pp. 998-1008, Jun. 1993.

[6] K. S. Pun, S. C. Chan, K. S. Yeung, and K. L. Ho, "On the design and implementation of FIR and IIR digital filters with variable frequency characteristics," IEEE Trans. Circuits Syst. II, Analog Digit. Signal Process., vol. 49, no. 11, pp. 689-703, Nov. 2002.

[7] P. Lowenborg and H. Johansson, "Linear programming design of linear-phase FIR filters with variable bandwidth," in Proc. IEEE ISCAS'2003, May 2003, vol. 3, pp. 554-557.

[8] K. M. Tsui, S. C. Chan, and K. W. Tse, "Design of complex-valued variable FIR digital filters and its application to the realization of arbitrary sampling rate conversion for complex signals," IEEE Trans. Circuits Syst. II, Analog Digit. Signal Process., vol. 52, no. 7, pp. 424-428, Jul. 2005

[9] R. Zarour and M. M. Fahmy, "A design technique for variable digital filters,” IEEE Trans. Circuits Syst., vol. 36, pp. 1473-1478, Nov. 1989.

[10] M. Makundi, T. I. Laakso, and V. Valimaki, "Efficient tunable IIR and allpass filter structures," IET Electron. Lett., vol. 37, pp. 344-345, Mar. 2001.

[11] T. B. Deng, "Design of recursive 1-D variable filters with guaranteed stability," IEEE Trans. Circuits Syst. II, Analog Digit. Signal Process., vol. 9, pp. 689-695, Sep. 1997.

[12] H. Zhao and H. K. Kwan, "Design of 1-D stable variable fractional delay IIR filters," in Proc. IEEE ISPACS, Dec. 2005, pp. 517-520.

[13] V. Valimaki and T. I. Laakso, "Suppression of transients in variable recursive digital filters with a novel and efficient cancellation method," IEEE Trans. Signal Process., vol. 46, pp. 3408-3414, Dec. 1998.

[14] H. Brandenstein and R. Unbehauen, "Least-squares approximation of FIR by IIR filters," IEEE Trans. Signal Process., vol. 46, no. 1, pp. 21-30, Jan. 1998

[15] S. C. Chan, K. M. Tsui, and K. W. Tse, "Design of constrained causal stable IIR filters using a new second order cone programming based model reduction technique," IEEE Trans. Circuits Syst. II, Exp. Briefs, vol. 54, no. 2, pp. 107-111, Feb. 2007.

[16] A. G. Dempster and M. D. Macleod, "Use of minimum-adder multiplier blocks in FIR digital filters," IEEE Trans. Circuits Syst. II, Exp. Briefs, vol. 42, pp. 569-577, Sep. 1995.

[17] J. F. Sturm, "Using SeDuMi 1.02, a MATLAB toolbox for optimization over symmetric cones," Optim. Math. Softw., vol. 11-12, pp. 625-653, 1999. 